\title{
Bassa velocità di crescita e ritardo puberale, attenzione all'anamnesi
}

\author{
Brunetto Boscherini*, Patrizia del Balzo**, Maria Teresa Fonte*** \\ *Già ordinario di Pediatria, Università Tor Vergata, Roma; **Pediatra di famiglia, ASL RM1; \\ ***Pediatra di famiglia, ASL RM5
}

\begin{abstract}
Leonardo, 13 anni, vive male la condizione di vedersi più piccolo al confronto con i suoi compagni. Viene quindi in ambulatorio per una valutazione della crescita staturale e dello sviluppo puberale.
\end{abstract}

\section{La storia}

Nato a termine da gravidanza fisiologica, peso alla nascita $3,150 \mathrm{~kg}$. Sviluppo psico-motorio regolare. Nella linea paterna c'è familiarità per patologie autoimmunitarie: zio con artrite reumatoide, zia con Sindrome di Sjögren, nonna con lupus eritematoso sistemico (LES), cugina di primo grado celiaca. Statura padre cm 174, statura madre $170 \mathrm{~cm}$; bersaglio genetico $172 \mathrm{~cm}$.

La madre ha avuto il menarca a 12 anni, il padre ha presentato una pubertà tarda (15 anni).

La crescita di Leonardo è stata regolare fino all'età prepuberale; a 6 anni la statura ed il peso erano al $50^{\circ}$ centile. Il ragazzo pratica sport a livello agonistico ed è una promessa delle giovanili di una squadra di calcio di serie $A$, anche se negli ultimi mesi ha dovuto rallentare gli allenamenti perché accusa facile stancabilità. I genitori riferiscono, inoltre, che da circa un anno sia l'appetito che la crescita staturo-ponderale sono sensibilmente diminuiti e attribuiscono questi cambiamenti alla loro separazione conflittuale tuttora in corso.

\section{L'esame clinico}

Esame biettivo: aspetto armonico, statura $144 \mathrm{~cm}(-1,2 \mathrm{DS})$, peso $38,5 \mathrm{~kg}, \mathrm{BMI}$ 18,57 ( $25^{\circ}$ centile), genitali prepuberi (testicoli $3 \mathrm{ml}$, pubarca assente), stato nutrizionale discreto, modico pallore della cute e delle mucose, negativo l'esame dei vari organi e apparati. L'età ossea è ritardata: 11 anni e 6/12 rispetto all'età anagrafica di 13anni. In prima ipotesi la causa più probabile dello scarso accrescimento poteva essere un ritardo costituzionale di crescita e di pubertà, anche se non si poteva escludere che l'intenso sport agonistico potesse giocare un ruolo inibente sullo sviluppo puberale. Abbiamo pertanto consigliato un nuovo controllo clinico dopo 6-9 mesi ed una riduzione dell'attività sportiva.
Al successivo controllo (13 anni e 7 mesi) la statura era di $145,5 \mathrm{~cm}$, la velocità di crescita staturale (VC) risultava molto bassa $(2,45 \mathrm{~cm} /$ anno $=-5 \mathrm{DS})$ ed il peso era diminuito $(37,6 \mathrm{~kg}$ con un BMI di 17,7 pari al $10-25^{\circ}$ centile). Sviluppo puberale assente.

\section{Scarso accrescimento associato a mancato inizio della pubertà nel maschio. Come comportarsi?}

L'anamnesi permette di escludere possibili cause quali, ad esempio, lo sport agonistico intenso o l'anoressia nervosa, peraltro rara nel sesso maschile. Successivamente si deve considerare la causa più frequente, il Ritardo Costituzionale di Crescita e di Pubertà (RCCP).

In questi soggetti i valori ematici di testosterone, di FSH e LH sono compatibili con uno stadio prepuberale.

$\mathrm{Nel}$ caso in cui la VC sia molto rallentata, può essere sospettato un deficit acquisito di $G H$; in questi pazienti il peso è meno compromesso rispetto alla statura, anzi spesso tende ad aumentare; inoltre l'IGF-1 e la IGFBP3 sono molto bassi. La diagnosi è confermata dai test di stimolo farmacologico. Una bassa VC, con concomitante aumento del peso e assenza di pubertà si osservano nel bambino con ipotiroidismo acquisito, in genere secondario a tiroidite autoimmune. Nella fase iniziale i sintomi possono essere molto sfumati, l'età ossea di regola è ritardata.

Il TSH è elevato, l'FT4 basso, gli anticorpi antitiroide quasi sempre presenti.

Altre possibili cause di bassa VC e ritardo staturale sono la malattia celiaca e le patologie tumorali all'esordio. Soggetti con ipogonadismo di origine gonadica possono mostrare un rallentamento della VC associato ad assenza dei caratteri sessuali secondari con livelli prepuberali di testosterone ed aumento delle gonadotropine, specie dell'FSH.

Una causa rara di bassa VC e di ritardo puberale è infine la malattia infiammatoria cronica dell'intestino (MICI), che comprende principalmente due distinte entità, la malattia di Crohn (MC) e la Colite Ulcerosa (CU). La diagnosi di queste condizioni viene spesso formulata con un ritardo di
1-2 anni dall'insorgenza dei primi sintomi per l'aspecificità delle modalità di presentazione.

\section{Ritorniamo al nostro caso}

In base all'anamnesi familiare positiva per pubertà tarda, alla bassa VC associata al mancato inizio della pubertà, al ritardo della maturazione scheletrica e alla normalità degli esami richiesti abbiamo sospettato un RCCP, ma dopo circa 4 mesi dal nostro ultimo controllo il ragazzo ha presentato un episodio caratterizzato da diarrea profusa, dolori addominali ed enterorragia: ricoverato in Gastroenterologia la diagnosi di dimissione è stata CU.

\section{Il sospetto diagnostico di MICl nell'ambulatorio pediatrico}

La presentazione clinica è fortemente influenzata dalla sede e dalla estensione del processo flogistico. In genere il sospetto diagnostico sorge in seguito alla comparsa di importanti sintomi gastrointestinali, come dolore addominale, diarrea sanguinolenta o mucosa e, in caso di localizzazione ileo-cecale, presenza di una massa addominale palpabile nel quadrante inferiore destro; sintomi che possono essere associati a ritardo di crescita e stato nutrizionale scadente. Mentre la CU esordisce più frequentemente con diarrea mucoematica e dolore addominale, nella MC oltre ai dolori addominali è prevalente il calo ponderale.

In generale, il ruolo degli esami di laboratorio è modesto; tuttavia, un valore di calprotectina fecale normale sembra essere sufficiente per escludere la diagnosi. Spesso negli anni precedenti la diagnosi di MI$\mathrm{CI}$, la $\mathrm{VC}$ è rallentata, in particolare nei pazienti con MC (60-90\%) ed in misura inferiore in quelli con CU (10-25\%). Alla diagnosi una bassa statura si osserva nel 6-10\% dei pazienti con MC; mentre nella $\mathrm{CU}$ in una minoranza dei pazienti $(3 \%)$. Se la MICI si manifesta in età puberale è comune osservare un ritardato inizio della pubertà o un rallentamento della progressione dei caratteri puberali secondario ad un ipogonadismo centrale transitorio, l'età ossea è ritardata (di circa 1-2 anni) in quasi la metà dei pazienti. Pertanto, questi pa- 


\begin{tabular}{l}
\hline \multicolumn{2}{|c|}{ TABELLA 1. Manifestazioni extraintestinali negli anni precedenti } \\
la diagnosi (da voce bibliografica 4, modificata)
\end{tabular}

Eccezionali $(<1 \%)$ : colangite primaria sclerosante, pancreatite, epatite cronica attiva, irite 0 uveite, spondilite anchilosante, pioderma gangrenoso.

zienti possono essere erroneamente considerati affetti da RCCP. Nel periodo che precede le gravi manifestazioni intestinali la difficoltà di sospettare la MICI è documentata dalla letteratura che indica un ritardo della diagnosi di 1-2 anni; tuttavia è importante ricordare che, oltre al rallentamento della crescita, in questo periodo possono essere già presenti sintomi intestinali minori (dolori addominali ricorrenti, episodi transitori di diarrea) oppure, più raramente, sintomi extraintestinali (Tabella 1).

La presenza di questi sintomi, che possono sfuggire se non intenzionalmente ricercati, può essere utile per indurre il sospetto diagnostico di MICI. Gli esami di routine (VES, PCR, fibrinogeno, ferritina, albumina, transaminasi, emocromo con conta piastrinica) sono di poco aiuto in quanto aspecifici. Anche se alcuni studi suggeriscono che la calprotectina fecale possa essere considerata un biomarker che permette di differenziare la MICI da altre patologie intestinali, tuttavia, data la bassa sensibilità e specificità, non può essere considerata attualmente una alternativa alla colonscopia, che resta il gold standard per la diagnosi di MICI.

\section{Rivalutazione critica del nostro caso}

Dopo la formulazione della diagnosi definitiva di CU da parte del gastroenterologo, abbiamo voluto rivalutare criticamente il nostro caso, allo scopo di evidenziare eventuali elementi sfuggiti alla nostra osservazione. In effetti, è emerso che dall'età di circa 11 anni il bambino aveva presentato alcuni episodi di dolore addominale di modesta entità e diarrea mucosa di breve durata. Per tale motivo, furono eseguiti alcuni esami, tra i quali due ecografie addominali, tutti con esito negativo. Inoltre, tra gli 11 e 12 anni erano comparsi 5-6 episodi di stomatite aftosa. Questi nuovi dati anamnestici, emersi solo con la rivalutazione del caso, potevano farci sospettare una MICI.

\section{Cosa abbiamo imparato}

In un bambino che in età puberale presenta scarso accrescimento staturale, ponderale o di entrambi, associato a mancata comparsa dei caratteri puberali, si deve sempre escludere la possibilità, seppur rara di MICI.

E necessario pertanto ricercare mediante una anamnesi mirata eventuali saltuari episodi di sintomi intestinali anche poco rilevanti oppure manifestazioni extraintestinali (soprattutto artralgie e stomatite aftosa); la loro esistenza giustifica il sospetto di MICI. Al contrario, la richiesta di esami di laboratorio non è necessaria, infatti i risultati che si ottengono, in quanto aspecifici, sono in genere di scarso aiuto.

brunetto_boscherini@fastwebnet.it

1. Heida A, Holtman GA, Lisman-van Leeuwen $\mathrm{Y}$, et al. Avoid Endoscopy in Children With Suspected Inflammatory Bowel Disease Who Have Normal Calprotectin Levels. J Pediatr Gastroenterol Nutr. 2016 Jan;62(1):4749.

2. Kanof ME, Lake AM, Bayless TM. Decreased height velocity in children and adolescents before the diagnosis of Crohn's disease. Gastroenterology. 1988 Dec;95(6):1523-157.

3. Jose FA, Garnett EA, Vittinghoff E, et al. Development of extraintestinal manifestations in pediatric patients with inflammatory bowel disease. Inflamm Bowel Dis. 2009 Jan;15(1):63-68.

4. Khaki-Khatibi F, Qujeq D, Kashifard M, et al. Calprotectin in inflammatory bowel disease. Clin Chim Acta. 2020 Nov;510:556565. 